\title{
Determinants of Change in Glycemic Status in Individuals with Prediabetes: Results from a Nationwide Cohort Study in Germany
}

\author{
Rebecca Paprott $(\mathbb{D}$, Christa Scheidt-Nave, and Christin Heidemann (D) \\ Department of Epidemiology and Health Monitoring, Robert Koch Institute, 12101 Berlin, Germany \\ Correspondence should be addressed to Christin Heidemann; heidemannc@rki.de
}

Received 18 April 2018; Revised 2 July 2018; Accepted 29 August 2018; Published 14 October 2018

Academic Editor: Paolo Fiorina

Copyright (c) 2018 Rebecca Paprott et al. This is an open access article distributed under the Creative Commons Attribution License, which permits unrestricted use, distribution, and reproduction in any medium, provided the original work is properly cited.

\begin{abstract}
Previous studies investigating determinants of changes in glycemic status among individuals with prediabetes mainly focused on glucose-defined prediabetes. In this study, we examined determinants of a regression to normoglycemia or a progression to diabetes among individuals with HbAlc-defined prediabetes. The study included 817 participants (18-79 years) with prediabetes (HbAlc 5.7-6.4\% $(39-47 \mathrm{mmol} / \mathrm{mol})$ ) at baseline. Glycemic status at follow-up was categorized as diagnosed diabetes (self-reported physician diagnosis or antidiabetic medication), undiagnosed diabetes (HbAlc $\geq 6.5 \%(\geq 48 \mathrm{mmol} / \mathrm{mol})$ ), prediabetes (as defined at baseline), and normoglycemia ( $\mathrm{HbAlc}<5.7 \%(<39 \mathrm{mmol} / \mathrm{mol}))$. Determinants of glycemic changes were identified by multinomial logistic regression (OR $(95 \% \mathrm{CI})$ ), with those remaining in the prediabetic state as reference. During a mean follow-up time of 12.0 years, $33.8 \%$ of the participants reverted to normoglycemia, $7.2 \%$ progressed to undiagnosed diabetes, $12.8 \%$ progressed to diagnosed diabetes, and $46.2 \%$ remained prediabetic. Determinants of a regression to normoglycemia were female sex (male vs. female: $0.67(0.46 ; 0.98)$ ) and higher HDL cholesterol levels $(1.17$ (1.02; 1.35$)$ per $10 \mathrm{mg} / \mathrm{dl})$. Determinants of a progression to undiagnosed or diagnosed diabetes were higher values of BMI (1.10 (1.02; 1.18); $1.13(1.06 ; 1.21)$ per $\left.\mathrm{kg} / \mathrm{m}^{2}\right)$, waist circumference $(1.04(1.01 ; 1.07) ; 1.06(1.03 ; 1.09)$ per $\mathrm{cm})$, alanine aminotransferase $(1.06$ $(1.03 ; 1.09) ; 1.07(1.03 ; 1.10)$ per U/l), and gamma-glutamyl transferase $(1.02(1.00 ; 1.03) ; 1.03(1.01 ; 1.04)$ per U/l). Higher age $(1.04(1.02 ; 1.06)$ per year), female sex (male vs. female: $0.56(0.33 ; 0.97))$, and parental history of diabetes (yes vs. no: 1.82 $(1.05 ; 3.15))$ were further associated with a progression to diagnosed diabetes, whereas higher triglyceride levels (1.03 (1.01; 1.06) per $10 \mathrm{mg} / \mathrm{dl}$ ) were associated with a progression to undiagnosed diabetes. In conclusion, among the investigated determinants, potentially modifiable anthropometric and metabolic markers were associated with glycemic changes in individuals with HbAlc-defined prediabetes. The findings of this study demonstrate the need for more refined case finding strategies for diabetes prevention.
\end{abstract}

\section{Introduction}

Type 2 diabetes is a chronic metabolic disease characterized by elevated blood glucose levels that increase the risk for severe micro- and macrovascular complications [1]. Prediabetes is considered a prestage of manifest diabetes and is associated with an increased risk of developing type 2 diabetes [2-5]. Approximately 5\% to $10 \%$ of individuals with prediabetes progress to diabetes yearly [2]. However, progression can be prevented by lifestyle and pharmacological interventions and prediabetes may even revert to normoglycemia $[2,3]$.
Prediabetes can be defined by impaired fasting glucose (IFG) $(5.6-6.9 \mathrm{mmol} / \mathrm{l})$, impaired glucose tolerance (IGT) (7.8-11.0 mmol/l), or elevated HbAlc levels (5.7-6.4\% (39$47 \mathrm{mmol} / \mathrm{mol}$ )) [5]. Previous studies on determinants of progression or regression from prediabetes were mostly based on prediabetes defined by IFG or IGT, whereas studies using elevated $\mathrm{HbAlc}$ levels for definition are sparse.

In these studies, among individuals with IFG or IGT, measures of lipid metabolism [6-10] and anthropometry $[4,8,11-14]$ were associated with changes in glycemic status. Moreover, several previous studies among persons with IFG or IGT examined the effect of modifiable lifestyle 
factors (mostly physical activity, smoking, and alcohol intake) on glycemic changes but the majority did not find an association [4, 6-10, 15-17]. In addition, a recent study that included individuals with HbAlc-defined prediabetes found no association between physical activity and a regression to normoglycemia [17].

Since the characteristics of individuals with prediabetes who are diagnosed by different criteria may vary due to differences in the underlying pathophysiological pathways $[2,18]$, determinants of changes in glycemic status may also differ according to the applied diagnostic criterion.

In the present study, we therefore aimed to identify determinants of a regression to normoglycemia or a progression to undiagnosed or diagnosed diabetes in a nationwide sample of German adults with $\mathrm{HbAlc}$-defined prediabetes who were followed for 12 years. In addition to sociodemographic characteristics, we investigated potentially modifiable risk factors such as anthropometric, metabolic, and lifestyle factors as determinants of change in glycemic status.

\section{Materials and Methods}

2.1. National Health Examination Surveys. The "German National Health Interview and Examination Survey 1998" (GNHIES98) was conducted from 1997 to 1999 and targeted the residential German population aged 18 to 79 years (response: 61\%). A two-stage cluster sampling procedure was applied. First, sample points were selected, and then, age- and sex-stratified random samples from local population registries were drawn [19]. Out of all GNHIES98 participants, 3959 participants (response: 62\%) also took part in the subsequent "German Health Interview and Examination Survey for Adults” (DEGS1), conducted from 2008 to 2011 (Figure 1) [20]. Both surveys were conducted in accordance with the Declaration of Helsinki and approved by the Federal and State Commissioners for Data Protection. DEGS1 was approved by the ethics committee of the CharitéUniversitätsmedizin Berlin (No. EA2/047/08). Participants provided written informed consent before participation [20].

2.2. Study Population. Figure 1 depicts a flow chart reflecting the composition of the study population. After applying different exclusion criteria among those who participated in both GNHIES98 (baseline) and DEGS1 (follow-up), a cohort consisting of 2900 participants remained. The final study sample comprised 817 participants with prediabetes at baseline.

2.3. Assessment of Glycemic Status. Diagnosed diabetes was determined by a self-reported history of diagnosed diabetes assessed in physician-administered standardized interviews or by the intake of antidiabetic agents within the 7 days prior to the interview documented through a detailed medication review. Among participants without diagnosed diabetes, normoglycemia ( $\mathrm{HbAlc}<5.7 \%(<39 \mathrm{mmol} / \mathrm{mol}))$, prediabetes (HbA1c 5.7-6.4\% (39-47 mmol $/ \mathrm{mol})$ ), and undiagnosed diabetes $(\mathrm{HbA} 1 \mathrm{c} \geq 6.5 \%(\geq 48 \mathrm{mmol} / \mathrm{mol}))$ were defined according to recent ADA recommendations [5]. In GNHIES98, HbAlc was measured using a Diamat high-performance liquid chromatography (HPLC) analyzer (Bio-Rad Laboratories, Munich, Germany) and RECIPE reagents (RECIPE Chemicals and Instruments, Munich, Germany). In DEGS1, HbAlc was measured using an immunoturbidimetric method (ARCHITECT ci8200; Abbott, Wiesbaden, Germany). Both methods were traceable to the National Glycohemoglobin Standardization Program [21], and no systematic deviation of HbAlc measurements between the surveys was evident [22].

2.4. Assessment of Risk Factors for Type 2 Diabetes. The assessment of several health-related risk factors in GNHIES98 has been previously described in detail [20, 22-25]. Briefly, information about educational level, sport activity, smoking status, residential traffic intensity, and mental distress was obtained through a standardized self-administered questionnaire. For the current analyses, middle and high educational levels were combined. Sport activity was ascertained as regular sport activity over the past three months and categorized as either no sport or any sport. Smoking status was categorized into never, former, and current smoker. Residential traffic intensity was dichotomized into rare to considerable traffic versus heavy to extreme traffic. Psychosocial distress was measured by the Mental Health Inventory (MHI-5) on a scale ranging from 0 to 100 points, with lower values indicating mental distress and higher values indicating mental well-being. A parental history of diabetes was assessed in DEGS1 through a standardized physician-administered personal interview. In GNHIES98, measures of body height, weight, and waist circumference were obtained while participants wore light clothing. BMI was calculated as the ratio of body weight $(\mathrm{kg})$ and height squared $\left(\mathrm{m}^{2}\right)$.

The intake of coffee, red meat, and whole grains (including whole grain bread, buns, and muesli) (as components of the German Diabetes Risk Score (GDRS) that is described below) was assessed through a questionnaire on frequency of consumption of these food groups. The average amount consumed per day was estimated by further considering information on dietary intake assessed through a diet history method in a GNHIES98 subsample $(n=4030)$ as previously described [23].

As a summary measure of overall risk for type 2 diabetes, we calculated the GDRS including age, height, waist circumference, history of hypertension, physical activity, smoking, family history of diabetes, and intake of coffee, red meat, and whole grains as score components [26]. The GDRS has been shown to be a valid tool for prediction of diagnosed diabetes in the general adult population [23].

Venous blood samples were taken, processed within an hour, and stored at $-40^{\circ} \mathrm{C}$ in the central laboratory unit at the Robert Koch Institute until further analyses. HDL cholesterol was determined based on the cholesterol oxidaseperoxidase 4-aminophenazone method (MEGA, Merck, Darmstadt, Germany) (intra-assay CV: 1.2-2.5\%). Triglycerides were determined by the glycerol-3-phosphate oxidaseperoxidase 4-aminophenazone method using the MEGA measurement device (Merck, Darmstadt, Germany) (intraassay CV: $1.6-2.2 \%)$. Both gamma-glutamyl transferase 


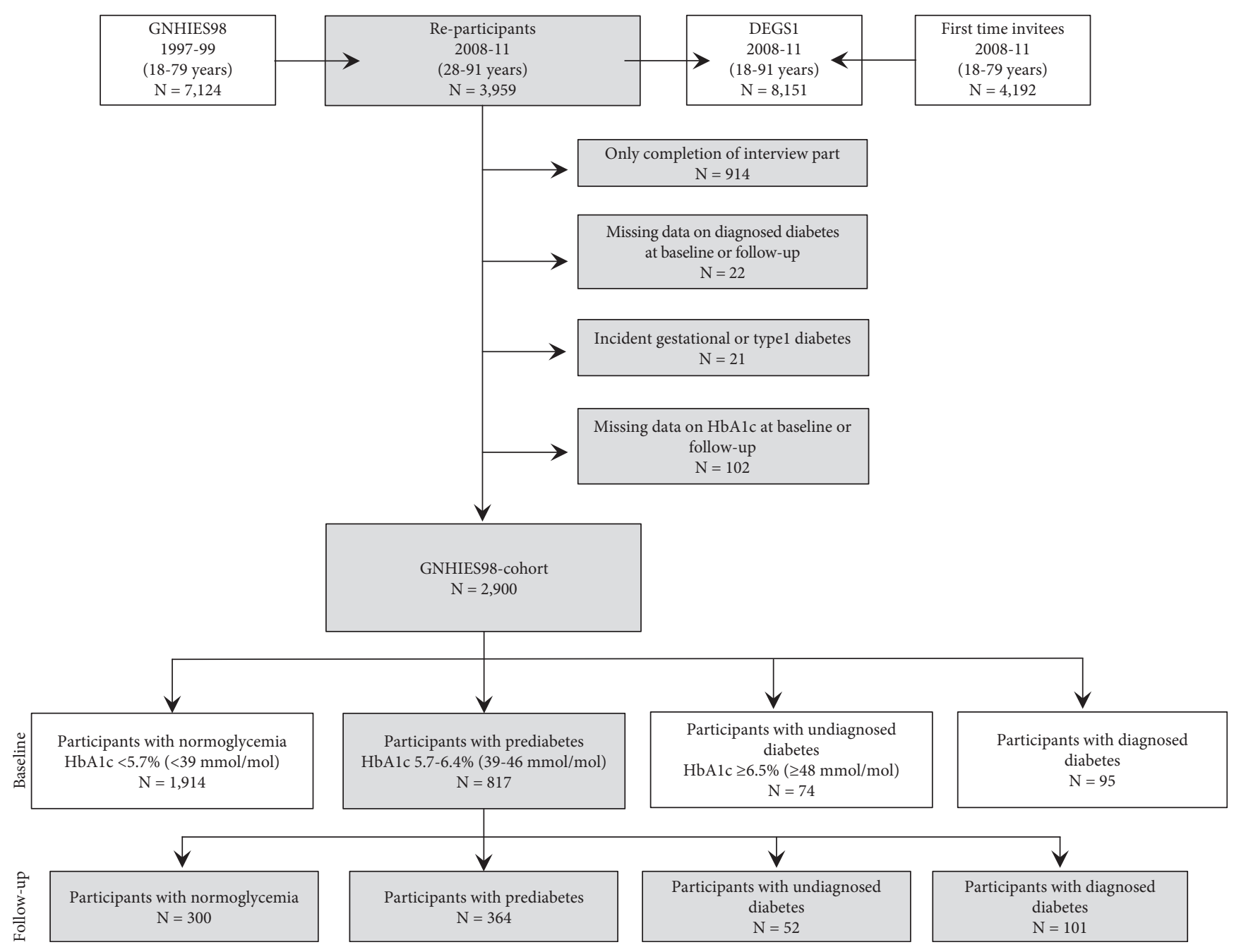

FIgURe 1: Flow chart showing the definition and distribution of participants within categories of glycemic status. Definitions applied for glycemic status were the same at baseline and follow-up. The numbers shown were drawn from the unimputed data set.

(GGT) and alanine aminotransferase (ALT) were measured according to standards of the DGKC (German Association of Clinical Chemistry) (EPOS, Eppendorf, Wesseling, Germany) (intra-assay CV for GGT: $1.3-2.7 \%$; for ALT: $1.1-$ $4.8 \%$ ). High-sensitivity C-reactive protein (hs-CRP) was measured in serum by an immunoturbidimetric method (ARCHITECT ci8200, Abbott, Munich, Germany) (intraassay CV: 4.0 ).

Additionally, diagnosed hypertension, hyperlipidemia, myocardial infarction, and stroke were considered as comorbidities and assessed through a physician-administered personal interview. The use of statins and thiazide diuretics was considered as concomitant therapy known to favor the progression from prediabetes to diabetes and was ascertained through a detailed medication review. However, we found that the number of participants taking thiazide diuretics $(n=20)$ or statins $(n=32)$ at the time of baseline examination (1997-99) was too low to enable further analyses.

2.5. Statistical Analysis. The SAS software 9.4 (SAS Institute Inc., Cary, NC) was used for all statistical analyses. A weighting factor was applied that corrects for deviations of the DEGS1 sample from population figures from the Federal Statistical Office (as of 31 December 2010) and accounts for the incomplete follow-up [27]. For the current analyses, the probability of GNHIES98 participants with prediabetes participating in DEGS1 was derived from a generalized linear mixed model with age at the time of GNHIES98 (4 categories) and age at the time of DEGS1 (8 categories), as well as smoking (yes or no), education (3 categories), income (3 categories), and migration background (yes or no) at the time of GNHIES98, as independent variables.

Multinomial logistic regression was performed to calculate the odds ratios (OR) and 95\% confidence intervals (CI) for considered risk factors. For the dependent variable, three categories were defined according to a participant's glycemic status at follow-up, with those who remained in the prediabetic state as the reference group. In the case of a regression to normoglycemia, an OR $>1.00$ indicates a higher chance for a regression (i.e., favorable). In the case of a progression to diabetes, an OR $>1.00$ indicates a higher chance of a progression (i.e., unfavorable). 
TABLe 1: Baseline characteristics of individuals with prediabetes at baseline $(n=817)$ according to HbAlc-defined glycemic status at follow-up.

\begin{tabular}{|c|c|c|c|c|}
\hline & \multirow[b]{2}{*}{$\begin{array}{l}\text { Regression to } \\
\text { normoglycemia }\end{array}$} & \multirow[b]{2}{*}{$\begin{array}{l}\text { Remained } \\
\text { prediabetic }\end{array}$} & \multicolumn{2}{|c|}{ Progression to } \\
\hline & & & $\begin{array}{l}\text { Undiagnosed } \\
\text { diabetes }\end{array}$ & $\begin{array}{c}\text { Diagnosed } \\
\text { diabetes }\end{array}$ \\
\hline$n(\%)$ & $300(33.8 \%)$ & $364(46.2 \%)$ & $52(7.2 \%)$ & $101(12.8 \%)$ \\
\hline \multicolumn{5}{|l|}{ Unmodifiable risk factors } \\
\hline \multicolumn{5}{|l|}{ Sociodemographic factors } \\
\hline Age (years) & $48.5(45.9 ; 51.1)$ & $49.6(47.6 ; 51.7)$ & $53.8(50.5 ; 57.2)$ & $57.0(54.8 ; 59.2)$ \\
\hline Male sex & $53.3(46.5 ; 60.1)$ & $61.5(54.8 ; 67.8)$ & $48.1(30.4 ; 66.2)$ & $41.6(31.1 ; 52.9)$ \\
\hline \multicolumn{5}{|l|}{ Educational level } \\
\hline Low & $52.2(45.0 ; 59.4)$ & $52.6(45.6 ; 59.6)$ & $52.4(34.2 ; 70.2)$ & $71.8(61.6 ; 82.0)$ \\
\hline Medium/high & $47.8(40.6 ; 55.0)$ & $47.4(40.4 ; 54.4)$ & $47.3(29.8 ; 65.8)$ & $28.2(18.0 ; 38.4)$ \\
\hline \multicolumn{5}{|l|}{ Family history of diabetes } \\
\hline At least one parent with diabetes & $27.1(19.6 ; 34.5)$ & $35.4(29.9 ; 40.8)$ & $31.1(10.6 ; 51.6)$ & $46.7(34.6 ; 58.8)$ \\
\hline \multicolumn{5}{|l|}{ Modifiable risk factors } \\
\hline \multicolumn{5}{|l|}{ Anthropometric markers } \\
\hline BMI $\left(\mathrm{kg} / \mathrm{m}^{2}\right)$ & $26.6(25.8 ; 27.3)$ & $27.5(27.0 ; 28.1)$ & $29.6(28.5 ; 30.6)$ & $30.4(29.3 ; 31.6)$ \\
\hline Waist circumference $(\mathrm{cm})$ & $91.1(89.1 ; 93.1)$ & $94.3(92.8 ; 95.7)$ & $98.6(94.7 ; 102.5)$ & $101.3(98.0 ; 104.5)$ \\
\hline \multicolumn{5}{|l|}{ Lifestyle factors } \\
\hline Any sport activity per week & $44.9(37.5 ; 52.4)$ & $51.2(44.3 ; 58.1)$ & $43.8(29.1 ; 59.9)$ & $39.6(29.8 ; 49.4)$ \\
\hline \multicolumn{5}{|l|}{ Smoker at baseline } \\
\hline Never & $36.9(30.4 ; 43.4)$ & $38.2(31.4 ; 45.0)$ & $51.2(36.5 ; 65.9)$ & $43.9(33.1 ; 54.7)$ \\
\hline Former & $16.7(11.7 ; 21.7)$ & $24.7(19.7 ; 29.7)$ & $26.7(15.6 ; 42.1)$ & $25.2(15.7 ; 34.8)$ \\
\hline Current & $46.4(39.1 ; 53.8)$ & $37.1(30.9 ; 43.3)$ & $21.8(11.9 ; 36.7)$ & $30.9(20.7 ; 41.1)$ \\
\hline Intake of whole grains (portions/day) & $1.3(1.2 ; 1.5)$ & $1.2(1.1 ; 1.4)$ & $1.0(0.8 ; 1.2)$ & $1.1(1.0-1.3)$ \\
\hline Intake of red meat (portions/day) & $0.58(0.55 ; 0.60)$ & $0.59(0.57 ; 0.61)$ & $0.54(0.50 ; 0.58)$ & $0.52(0.47-0.57)$ \\
\hline Intake of coffee (cups/day) & $3.2(2.9 ; 3.4)$ & $3.1(2.8 ; 3.3)$ & $2.8(2.4 ; 3.1)$ & $2.8(2.4 ; 3.2)$ \\
\hline \multicolumn{5}{|l|}{ Residential traffic intensity } \\
\hline $\begin{array}{l}\text { Heavy/extreme residential traffic } \\
\text { intensity }\end{array}$ & $25.5(18.3 ; 32.7)$ & $23.5(17.7 ; 29.4)$ & $28.1(13.5 ; 49.6)$ & $33.5(22.8 ; 44.2)$ \\
\hline Mental distress (points) & $73.9(71.6 ; 76.1)$ & $75.4(73.3 ; 77.4)$ & $79.0(74.2 ; 83.7)$ & $70.9(67.0 ; 74.8)$ \\
\hline GDRS (points) & $538(514 ; 563)$ & $575(557 ; 592)$ & $633(599 ; 666)$ & $690(661 ; 720)$ \\
\hline \multicolumn{5}{|l|}{ Metabolic markers } \\
\hline HDL cholesterol (mg/dl) & $56.4(53.5 ; 59.4)$ & $52.4(50.4 ; 54.6)$ & $51.3(47.9 ; 54.9)$ & $50.3(47.5 ; 53.2)$ \\
\hline Triglycerides (mg/dl) & $121.9(111.0 ; 133.8)$ & $144.1(134.2 ; 154.7)$ & $187.0(150.2 ; 232.9)$ & $\begin{array}{c}175.2(155.0 \\
198.0)\end{array}$ \\
\hline GGT (U/l) & $13.9(12.7 ; 15.1)$ & $15.6(14.6 ; 16.7)$ & $18.9(16.2 ; 22.1)$ & $20.1(17.2 ; 23.5)$ \\
\hline $\operatorname{ALT}(\mathrm{U} / \mathrm{l})$ & $12.3(11.4 ; 13.2)$ & $12.7(12.0 ; 13.5)$ & $16.0(13.3 ; 19.2)$ & $15.0(13.5 ; 16.5)$ \\
\hline hs-CRP (mg/l) & $1.4(1.1 ; 1.7)$ & $1.3(1.2 ; 1.5)$ & $1.4(0.9 ; 2.3)$ & $2.3(1.7 ; 3.1)$ \\
\hline
\end{tabular}

Information is given as weighted proportions $(95 \% \mathrm{CI})$ for categorical variables, as weighted arithmetic mean $(95 \% \mathrm{CI})$ for continuous variables, and in case of metabolic markers and predicted 5-year diabetes risk as weighted geometric mean (95\% CI). Differences in proportions and means between groups of glycemic status (reference group: remained prediabetic) were assessed by logistic regression and ANOVA. Bold numbers indicate $p<0.05$.

We classified a total of 19 potential determinants (Table 1) into the following categories and subcategories: (1) unmodifiable diabetes risk factors comprising sociodemographic factors and family history of diabetes and (2) potentially modifiable risk factors, including anthropometric markers, lifestyle factors, residential traffic intensity, mental distress, the diabetes risk score (as a summary measure of predominantly modifiable factors), and metabolic markers. Risk factors were examined in separate multinomial regression models, adjusting for age and sex (model 1). For potentially modifiable risk factors, a second model (model 2) was fitted that additionally adjusted for educational level and other potential confounders that were selected by applying directed acyclic graphs [28].

Among individuals with prediabetes at baseline, $22 \%$ had missing data in at least one of the considered determinants. To account for the missing values, multiple imputation was applied that included all potential determinants, glycemic 
status at follow-up, the weighting factor, and the cluster variable. The SAS procedure "PROC MI" with the fully conditional specification method for an arbitrary pattern of missingness was used, and 25 complete data sets were imputed $[29,30]$. The fraction of missing information ranged from $<0.001$ for height to 0.08 for hs-CRP. The relative efficiency was $>0.99$ for all risk factors imputed. SAS survey procedures were also applied to the individual imputed data sets again using the weighting factor and the cluster variable [29]. Results from the individual imputed data sets were then combined using the SAS procedure "PROC MIANALYZE," which accounts for the variability between the results of imputed data sets [31].

A considerable proportion of participants had an HbAlc level of $5.7 \%$ ( $39 \mathrm{mmol} / \mathrm{mol}$; equivalent to $26.1 \%$ ) or of $6.4 \%$ ( $47 \mathrm{mmol} / \mathrm{mol}$; equivalent to $2.4 \%$ ), i.e., an $\mathrm{HbAlc}$ level that was borderline for the definition of normoglycemia or diabetes, respectively. Therefore, relevant changes in glycemic status were defined for a sensitivity analysis as a change in the category of glycemic status combined with a change of at least \pm 0.3 percentage points in $\mathrm{HbAlc}$. This definition of a relevant glycemic change was adapted from Kowall et al., who defined relevant changes in glycemic status based on fasting or $2 \mathrm{~h}$ glucose [12].

In additional analyses, the associations between changes in modifiable risk factors during follow-up and changes in glycemic status were examined. A change in a modifiable risk factor was defined as the measurement at follow-up minus the measurement at baseline. In addition to models 1 and 2 described above, a third model (model 3) was analyzed additionally including the baseline value of the respective risk factor.

\section{Results}

During a mean follow-up time of 12.0 years, $7.2 \%$ of the 817 participants with prediabetes at baseline progressed to undiagnosed diabetes and $12.8 \%$ progressed to diagnosed diabetes. During the same period, $33.8 \%$ reverted to normoglycemia and $46.2 \%$ remained prediabetic (Table 1). When considering relevant changes in glycemic status, the proportion of participants who reverted to normoglycemia was $28.1 \%$ (95\% CI: $22.5-34.4 \%$ ), while $51.9 \%$ (46.2$57.6 \%)$ remained in the prediabetic stage. The proportion of participants with a progression to undiagnosed or diagnosed diabetes remained unchanged (data not shown).

Baseline characteristics of participants according to changes in glycemic status during follow-up are shown in Table 1. Compared to those who remained in the prediabetic state, participants who regressed to normoglycemia had lower values for BMI, waist circumference, triglycerides, GGT, and the diabetes risk score. Additionally, they showed higher levels of HDL cholesterol. On the other hand, participants who progressed to diagnosed or undiagnosed diabetes were older and had higher values for BMI, waist circumference, triglycerides, GGT, ALT, and the diabetes risk score. Those who progressed to diagnosed diabetes were also more often female, living in streets with heavy or extreme residential traffic intensity, of a low educational level, and characterized by a lower intake of red meat and a higher level of hs-CRP. Those who progressed to undiagnosed diabetes were less likely to be current smokers and had a lower intake of whole grains compared to those who remained prediabetic.

Additional descriptive analyses considering diagnosed comorbidities at baseline (hypertension, hyperlipidemia, myocardial infarction, and stroke) showed that, as expected, participants with a progression to diagnosed diabetes were more likely and those with a regression to normoglycemia were less likely to have been diagnosed with hypertension or hyperlipidemia than those who remained prediabetic. No significant differences across categories of glycemic changes were observed with respect to myocardial infarction and stroke (data not shown).

Table 2 depicts the association between the investigated baseline determinants and changes in glycemic status as determined from multivariate analyses. Among the unmodifiable risk factors, female sex was associated with a regression to normoglycemia and a progression to diagnosed diabetes. Higher age and a parental history of diabetes were associated with a progression to diagnosed diabetes. Among the potentially modifiable risk factors, higher levels of HDL cholesterol and lower values of the diabetes risk score were associated with a regression to normoglycemia. Higher values of BMI, waist circumference, GGT, ALT, and the diabetes risk score were associated with a progression to both undiagnosed diabetes and diagnosed diabetes. In addition, a higher triglyceride level was associated with a progression to undiagnosed diabetes. The lifestyle factors considered, residential traffic intensity, and mental distress were not individually associated with a change in glycemic status. The results persisted when analyses were repeated considering only relevant glycemic changes (data not shown).

Further analyses of changes in potentially modifiable risk factors between baseline and follow-up (Supplementary Table 1) revealed that decreases in the levels of BMI (OR: 0.86 (95\% CI: 0.77-0.95)), waist circumference (0.96 (0.93$0.99)$ ), and triglycerides (0.94 (0.89-1.00)) during follow-up were associated with a regression to normoglycemia. However, an increased waist circumference (1.04 (1.00-1.08)) was associated with a progression to undiagnosed diabetes whereas an increased triglyceride level (1.05 (1.02-1.09)) was related to a progression to diagnosed diabetes. An increase in the diabetes risk score during follow-up was associated with a progression to undiagnosed diabetes (1.04 (1.01-1.08)) and to diagnosed diabetes (1.06 (1.01-1.10)).

\section{Discussion}

After 12.0 years of follow-up, 20\% of HbA1c-defined prediabetic adults from a nationwide German study had progressed to diagnosed or undiagnosed diabetes, whereas $46 \%$ had remained prediabetic and $34 \%$ had reverted to normoglycemia. Figure 2 provides an overview of the hypothesized determinants of the study and its findings. Among the unmodifiable risk factors, female sex was related to a higher chance of a regression to normoglycemia and a progression to diagnosed diabetes. Additionally, older age and a parental diabetes history were related to a higher chance of a progression to 


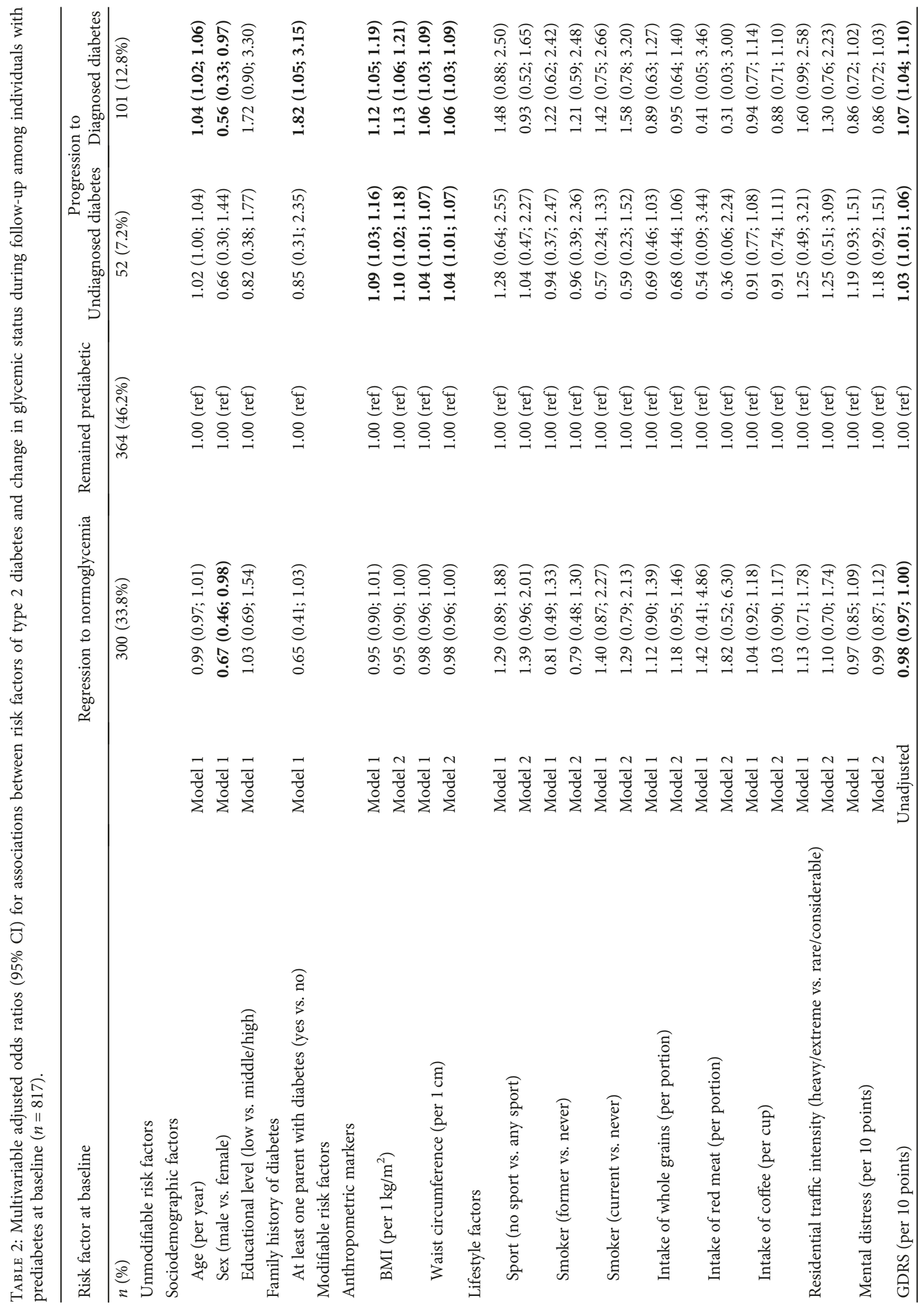




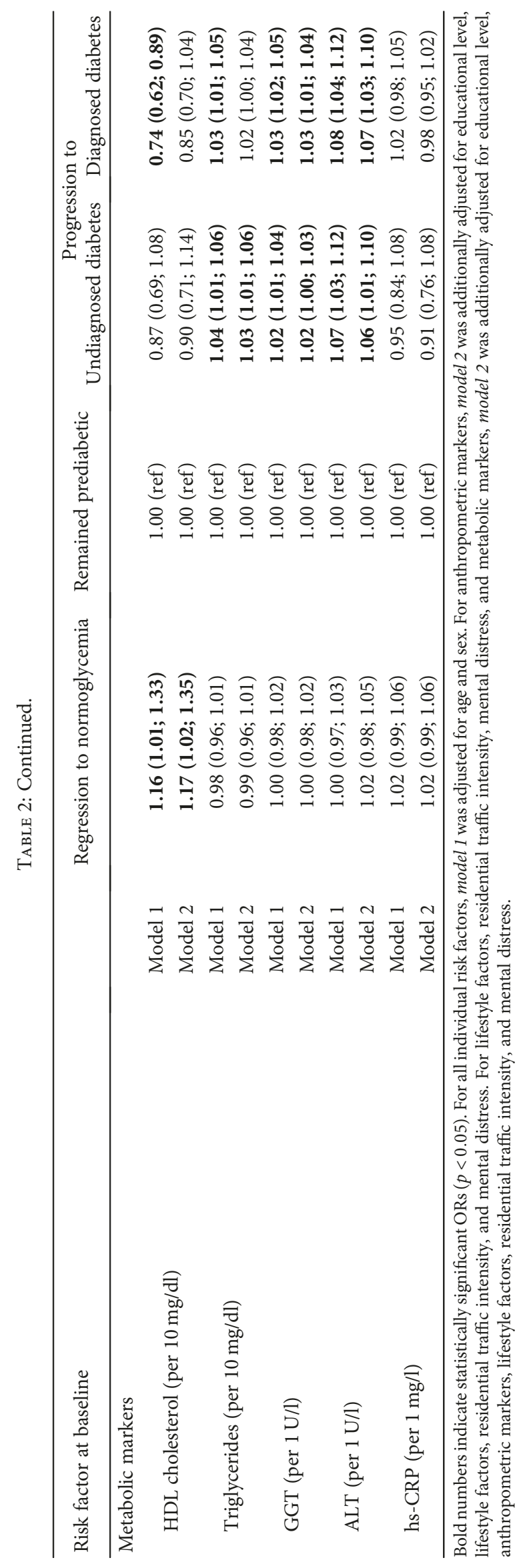




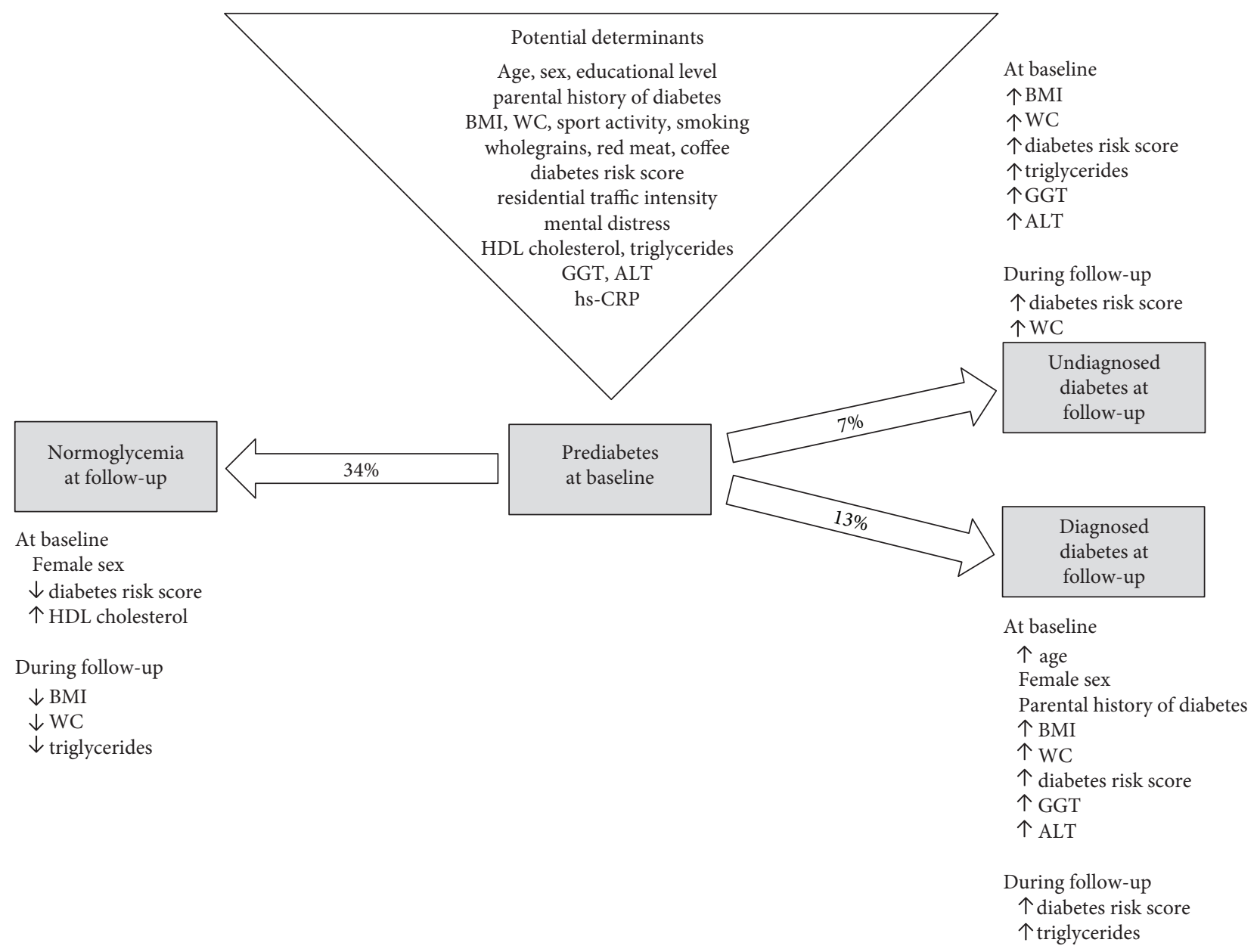

Figure 2: Overview on hypothesized determinants and study results. $\uparrow$ is equivalent to "higher at baseline/increase during follow-up"; $\downarrow$ is equivalent to "lower at baseline/decrease during follow-up"; BMI: body mass index; WC: waist circumference; HDL: high-density lipoprotein; GGT: gamma-glutamyl transferase; ALT: alanine aminotransferase; hs-CRP: high-sensitivity C-reactive protein.

diagnosed diabetes. Among potentially modifiable risk factors considered in the current study at baseline, favorable HDL cholesterol levels were related to a regression to normoglycemia. Less favorable levels of anthropometric markers and liver enzymes were associated with a progression to undiagnosed diabetes and diagnosed diabetes. In addition, less favorable triglyceride levels were related to a progression to undiagnosed diabetes. Higher values of the diabetes risk score as a summary measure of predominantly modifiable risk factors were associated with a progression to undiagnosed diabetes and diagnosed diabetes, whereas lower values were related to a regression to normoglycemia.

4.1. Discussion of Main Results. In this cohort, women had a higher chance of both a regression to normoglycemia and a progression to diagnosed diabetes. This finding might reflect a higher utilization of healthcare and greater health consciousness among women compared to men [32]. Higher age was associated with progression to diagnosed diabetes, confirming the well-known positive association between aging and the risk of type 2 diabetes. In addition, our findings related to a parental history of diabetes are consistent with findings from previous studies, indicating that individuals with a family history of diabetes are more likely to have their glucose level tested [33] and that a family history of diabetes probably affects an individual's knowledge of having diabetes [34].

Our findings regarding BMI and waist circumference mostly comport with findings from previous studies examining progression from IFG or IGT to diabetes $[8,11,13,14]$. In contrast with our findings, previous studies from India and South Africa did not detect an association between waist circumference and a progression to diabetes $[6,35]$. This disparity in results might be partly explained by the different ethnic groups included in the study populations. In our study, only decreases in BMI and waist circumference during follow-up but not their baseline values determined regression to normoglycemia. This finding is consistent with results of regional cohort studies of individuals with IFG or IGT in Germany [12] or the UK [4].

Interestingly, in multivariate analyses, none of the considered lifestyle factors was significantly associated with glycemic changes. This was also the case for fruit or vegetable intake and consumption of sugar-sweetened beverages or 
alcohol (data not shown). For physical activity, our null finding is consistent with the majority of previous studies that define glycemic status based on IFG, IGT $[4,6,7,9$, 10, 15, 17], or HbA1c [17]. Only a few prior studies have investigated the association between dietary behaviors and changes in glycemic status in individuals with prediabetes, and the results have been inconsistent $[7,16]$. The same is true for the association between smoking status and changes in glycemic status in individuals with prediabetes [6-10]. Differences in results might be explained by differences in the accuracy of the assessment methods used. In most epidemiological studies, including the present study, lifestyle factors are assessed through self-reporting, which is considered a rather imprecise method and is moreover prone to social desirability and recall bias [36, 37]. Given the high potential for misclassification, existing associations between lifestyle factors and change in glycemic status might not be detected [4]. In the case of physical activity, we also had to combine the assessed 5 categories into a dichotomous variable due to the relatively small sample sizes in certain categories, which might have been too broad to identify existing associations. Of particular relevance to dietary intake, a further explanation for our null finding might be that the influence of individual dietary components is fairly small and therefore difficult to detect. In the present study, values of the diabetes risk score had the expected associations with glycemic changes. These findings could not be fully explained by waist circumference or age (as two main score components) and thus highlight the value of a multifactorial diabetes risk assessment. In addition, a recent interventional study suggests that among individuals with prediabetes, there might be a high-risk phenotype that does not respond to lifestyle interventions such as dietary counseling and exercise [38-40].

Despite their discussed role in diabetes pathophysiology, traffic intensity and mental distress were also not related to glycemic changes among prediabetic adults in multivariate analyses. While we did not find a comparable study on traffic-related exposures, one study showed that mental loss as assessed by the mental component score of the SF-12 questionnaire was associated with a progression to diabetes in adults with IFG or IGT [41].

Similar to our findings, previous studies of individuals with IFG found higher baseline levels $[7,8,10]$ or an increase in triglycerides [7] to be associated with a progression in glycemic status. However, the results that show a progression in glycemic status are less consistent for studies that analyze individuals with IFG and IGT together [6, 9, 35]. Only a few previous studies have explored measures of lipid metabolism with respect to a regression in glycemic status from IFG or IGT, and the findings have been inconsistent $[9,10]$. The findings of the current study for liver enzymes, i.e., the association of higher GGT and ALT levels with progression in glycemic status, are in line with findings for GGT observed for individuals with IFG or IGT in a South African cohort [35]. With respect to hs-CRP, a study among Chinese adults with IGT found that higher levels of hs-CRP indicated a progression to diabetes [42], whereas no association with glycemic changes was observed in our study.
4.2. Limitations. This nationwide cohort study comprised participants from the general adult population in Germany covering a wide age range. However, the follow-up rate was rather moderate and the GNHIES98 participants who took part in the follow-up survey had some different characteristics from those who did not reparticipate or were deceased [24]. To account for differences arising from nonparticipation, a weighting factor was computed following the approach described above [27] and applied to all analyses in the present study.

Despite the application of a weighting factor, our study population might have been healthier compared to other studies. This was reflected by a higher proportion (34\%) of individuals who reverted to normoglycemia compared to some other studies. For example, the proportion of participants with a regression was $4.4 \%$ based on data from the Whitehall II study, which applied the same definition for prediabetes but had a shorter follow-up time [17]. On the other hand, similar to that in our study, the proportion of individuals with a progression to diabetes was approximately one-fifth of participants in the Atherosclerosis Risk in Communities (ARIC) study, which used the same prediabetes definition and a similar follow-up length [43] as those of our study. One reason for the rather high proportion of individuals who reverted to normoglycemia in our study might be the relatively high proportion of individuals at the lower threshold of HbAlc-defined prediabetes. Thus, in a sensitivity analysis, we applied a stricter definition to identify only relevant changes in glycemic status. Nonetheless, the results remained largely unchanged.

Moreover, the proportion of participants with a migration background was rather low, and therefore, ethnicity could not be taken into account as a potential determining factor for glycemic changes [44]. We also had no information on whether participants only temporarily progressed to diabetes or regressed to normoglycemia between baseline and follow-up [45]. Further, we cannot rule out that the observed associations between changes in risk factors and changes in glycemic status in the additional analyses might be biased from reverse causation. Moreover, we did not adjust for multiple testing since analyses were exploratory.

Based on $\mathrm{HbAlc}$ measures and information about diabetes diagnosis, the present study permitted the identification of similarities and differences in the progression to diagnosed and undiagnosed diabetes. However, due to the lack of additional markers of glucose metabolism, we were unable to directly compare findings between individuals whose prediabetes was based on HbAlc and those whose prediabetes was defined by glucose criteria (e.g., IFG or IGT). The definition of prediabetes based on either of these measures might be too narrow, and a more refined identification of subgroups at high risk of developing diabetes remains a challenge with high relevance for the development of effective case finding and prevention strategies $[38,39,46]$.

\section{Conclusion}

In this nationwide cohort of German adults who were followed for 12 years, approximately one-third of the 
individuals with $\mathrm{HbAlc}$-based prediabetes reverted to normoglycemia and one-fifth progressed to self-reported diagnosed or HbAlc-based undiagnosed diabetes. Measures of anthropometry and lipid metabolism at baseline or as change during follow-up showed the most consistent associations with changes in glycemic status in both directions. In addition, elevated liver enzymes were related to the future development of undiagnosed or diagnosed diabetes; older age and a family history of diabetes were further associated with future diagnosed diabetes. In contrast with results from previous studies that primarily relied on glucose-based definitions of prediabetic states, the findings of the current study appear to be more variable for metabolic than for anthropometric markers. This difference is not surprising, given that the use of different diagnostic criteria to define glycemic status is likely to represent different pathophysiological states of glucose dysregulation. Our results further support the relevance of the $\mathrm{HbAlc}$ criterion in the definition of prediabetes and also demonstrate the need for more refined case finding strategies for diabetes prevention.

\section{Abbreviations}

$\begin{array}{ll}\text { ADA: } & \text { American Diabetes Association } \\ \text { ALT: } & \text { Alanine aminotransferase } \\ \text { DEGS1: } & \begin{array}{l}\text { German Health Interview and Examination } \\ \text { Survey for Adults }\end{array} \\ \text { GGT: } & \text { Gamma-glutamyl transferase } \\ \text { GNHIES98: } & \text { German National Health Interview and } \\ & \text { Examination Survey 1998 } \\ \text { GDRS: } & \text { German Diabetes Risk Score } \\ \text { hs-CRP: } & \text { High-sensitivity C-reactive protein } \\ \text { IFG: } & \text { Impaired fasting glucose } \\ \text { IGT: } & \text { Impaired glucose tolerance. }\end{array}$

\section{Data Availability}

The data set used in the study cannot be made publicly available because informed consent from study participants did not cover public access to the data. However, the minimal data set underlying the findings presented in this study is archived in the "Health Monitoring" Research Data Centre at the Robert Koch Institute (RKI) and may be accessed by all interested researchers. On-site access to the data set is possible at the Secure Data Center of the RKI's "Health Monitoring" Research Data Centre. Requests should be submitted to fdz@rki.de.

\section{Conflicts of Interest}

The authors declare that there is no conflict of interest regarding the publication of this paper.

\section{Acknowledgments}

The survey GNHIES98 and the primary part of DEGS1 were funded by the German Federal Ministry of Health. This study was supported by research grants from the German Federal Ministry of Health (FKZ: GE20130320).

\section{Supplementary Materials}

Supplementary Table 1: multivariable adjusted odds ratios (95\% CI) for associations between change in modifiable risk factors of type 2 diabetes and change in glycemic status during follow-up among individuals with prediabetes at baseline $(n=817)$. (Supplementary Materials)

\section{References}

[1] J. S. Skyler, G. L. Bakris, E. Bonifacio et al., "Differentiation of diabetes by pathophysiology, natural history, and prognosis," Diabetes, vol. 66, no. 2, pp. 241-255, 2017.

[2] A. G. Tabak, C. Herder, W. Rathmann, E. J. Brunner, and M. Kivimaki, "Prediabetes: a high-risk state for diabetes development," Lancet, vol. 379, no. 9833, pp. 2279-2290, 2012.

[3] J. W. Stevens, K. Khunti, R. Harvey et al., "Preventing the progression to type 2 diabetes mellitus in adults at high risk: a systematic review and network meta-analysis of lifestyle, pharmacological and surgical interventions," Diabetes Research and Clinical Practice, vol. 107, no. 3, pp. 320331, 2015.

[4] D. H. Bodicoat, K. Khunti, B. T. Srinivasan et al., "Incident type 2 diabetes and the effect of early regression to normoglycaemia in a population with impaired glucose regulation," Diabetic Medicine, vol. 34, no. 3, pp. 396-404, 2017.

[5] American Diabetes Association, "2. Classification and diagnosis of diabetes: standards of medical care in diabetes - 2018," Diabetes Care, vol. 41, Supplement 1, pp. S13-S27, 2018.

[6] R. M. Anjana, C. S. Shanthi Rani, M. Deepa et al., "Incidence of diabetes and prediabetes and predictors of progression among Asian Indians: 10-year follow-up of the Chennai Urban Rural Epidemiology Study (CURES)," Diabetes Care, vol. 38, no. 8, pp. 1441-1448, 2015.

[7] S. S. Rasmussen, C. Glumer, A. Sandbaek, T. Lauritzen, and K. Borch-Johnsen, "Determinants of progression from impaired fasting glucose and impaired glucose tolerance to diabetes in a high-risk screened population: 3 year follow-up in the ADDITION study, Denmark," Diabetologia, vol. 51, no. 2, pp. 249-257, 2008.

[8] G. A. Nichols, T. A. Hillier, and J. B. Brown, "Progression from newly acquired impaired fasting glusose to type 2 diabetes," Diabetes Care, vol. 30, no. 2, pp. 228-233, 2007.

[9] X. Song, M. Qiu, X. Zhang et al., "Gender-related affecting factors of prediabetes on its 10-year outcome," BMJ Open Diabetes Research \& Care, vol. 4, no. 1, article e000169, 2016.

[10] L. de Abreu, K. L. Holloway, M. A. Kotowicz, and J. A. Pasco, "Dysglycaemia and other predictors for progression or regression from impaired fasting glucose to diabetes or normoglycaemia," Journal of Diabetes Research, vol. 2015, Article ID 373762, 8 pages, 2015.

[11] S. L. Edelstein, W. C. Knowler, R. P. Bain et al., "Predictors of progression from impaired glucose tolerance to NIDDM: an analysis of six prospective studies," Diabetes, vol. 46, no. 4, pp. 701-710, 1997.

[12] B. Kowall, W. Rathmann, M. Heier et al., "Impact of weight and weight change on normalization of prediabetes and on persistence of normal glucose tolerance in an older population: the KORA S4/F4 study," International Journal of Obesity, vol. 36, no. 6, pp. 826-833, 2011. 
[13] A. Gautier, R. Roussel, P. H. Ducluzeau et al., "Increases in waist circumference and weight as predictors of type 2 diabetes in individuals with impaired fasting glucose: influence of baseline BMI: data from the DESIR study," Diabetes Care, vol. 33, no. 8, pp. 1850-1852, 2010.

[14] A. F. Ishola, H. C. Gerstein, J. C. Engert et al., "Longitudinal relationships between glycemic status and body mass index in a multiethnic study: evidence from observational and genetic epidemiology," Scientific Reports, vol. 6, no. 1, article 30744, 2016.

[15] S. Engberg, C. Glumer, D. R. Witte, T. Jorgensen, and K. Borch-Johnsen, "Differential relationship between physical activity and progression to diabetes by glucose tolerance status: the Inter99 study," Diabetologia, vol. 53, no. 1, pp. 70-78, 2010.

[16] P. Lecomte, S. Vol, E. Cacès et al., "Five-year predictive factors of type 2 diabetes in men with impaired fasting glucose," Diabetes \& Metabolism, vol. 33, no. 2, pp. 140-147, 2007.

[17] K. Faerch, D. R. Witte, E. J. Brunner et al., "Physical activity and improvement of glycemia in prediabetes by different diagnostic criteria," The Journal of Clinical Endocrinology and Metabolism, vol. 102, no. 10, pp. 3712-3721, 2017.

[18] D. Vistisen, D. R. Witte, E. J. Brunner et al., "Risk of cardiovascular disease and death in individuals with prediabetes defined by different criteria: the Whitehall II study," Diabetes Care, vol. 41, no. 4, pp. 899-906, 2018.

[19] W. Thefeld, H. Stolzenberg, and B.-M. Bellach, "German National Health Interview and Examination Survey: Response, composition of participants, and analysis of non-respondents," Gesundheitswesen, vol. 61, pp. S57-S61, 1999.

[20] C. Scheidt-Nave, P. Kamtsiuris, A. Gosswald et al., "German Health Interview and Examination Survey for Adults (DEGS) - design, objectives and implementation of the first data collection wave," BMC Public Health, vol. 12, no. 1, p. 730, 2012.

[21] Diabetes Control and Complications Trial Research Group, D. M. Nathan, S. Genuth et al., "The effect of intensive treatment of diabetes on the development and progression of long-term complications in insulin-dependent diabetes mellitus," The New England Journal of Medicine, vol. 329, no. 14, pp. 977-986, 1993.

[22] C. Heidemann, Y. Du, R. Paprott, M. Haftenberger, W. Rathmann, and C. Scheidt-Nave, "Temporal changes in the prevalence of diagnosed diabetes, undiagnosed diabetes and prediabetes: findings from the German Health Interview and Examination Surveys in 1997-1999 and 2008-2011," Diabetic Medicine, vol. 33, no. 10, pp. 1406-1414, 2016.

[23] R. Paprott, K. Mühlenbruch, G. B. M. Mensink et al., "Validation of the German Diabetes Risk Score among the general adult population: findings from the German Health Interview and Examination Surveys," BMJ Open Diabetes Research \& Care, vol. 4, no. 1, article e000280, 2016.

[24] C. Heidemann, H. Niemann, R. Paprott, Y. Du, W. Rathmann, and C. Scheidt-Nave, "Residential traffic and incidence of type 2 diabetes: the German Health Interview and Examination Surveys," Diabetic Medicine, vol. 31, no. 10, pp. 1269-1276, 2014.

[25] M. J. Kelly, F. D. Dunstan, K. Lloyd, and D. L. Fone, "Evaluating cutpoints for the MHI-5 and MCS using the GHQ-12: a comparison of five different methods," BMC Psychiatry, vol. 8, no. 1, p. 10, 2008.
[26] K. Mühlenbruch, T. Ludwig, C. Jeppesen et al., "Update of the German Diabetes Risk Score and external validation in the German MONICA/KORA study," Diabetes Research and Clinical Practice, vol. 104, no. 3, pp. 459-466, 2014.

[27] P. Kamtsiuris, M. Lange, R. Hoffmann et al., "The first wave of the German health interview and examination survey for adults (DEGS1): sample design, response, weighting and representativeness," Bundesgesundheitsblatt Gesundheitsforschung Gesundheitsschutz, vol. 56, no. 5-6, pp. 620-630, 2013.

[28] S. Greenland, J. Pearl, and J. M. Robins, "Causal diagrams for epidemiologic research," Epidemiology, vol. 10, no. 1, pp. 3748, 1999.

[29] P. A. Berglund, "Multiple imputation using the fully conditional specification method: a comparison of SAS ${ }^{\circledR}$, Stata, IVEware, and R. Paper 2081-2015," 2015, http://support.sas.com/ resources/papers/proceedings15/2081-2015.pdf.

[30] T. E. Bodner, "What improves with increased missing data imputations?," Structural Equation Modeling: A Multidisciplinary Journal, vol. 15, no. 4, pp. 651-675, 2008.

[31] B. Ratitch, I. Lipkovic, and M. O'Kelly, Combining Analysis Results from Multiply Imputed Categorical Data, PharmaSUG 2013-Paper SP03, 2013.

[32] A. Oksuzyan, K. Juel, J. W. Vaupel, and K. Christensen, "Men: good health and high mortality. Sex differences in health and aging," Aging Clinical and Experimental Research, vol. 20, no. 2, pp. 91-102, 2008.

[33] H. J. Murff, R. L. Rothman, D. W. Byrne, and S. Syngal, “The impact of family history of diabetes on glucose testing and counseling behavior in primary care," Diabetes Care, vol. 27, no. 9, pp. 2247-2248, 2004.

[34] R. Valdez, P. W. Yoon, T. Liu, and M. J. Khoury, "Family history and prevalence of diabetes in the U.S. population: the 6year results from the National Health and Nutrition Examination Survey (1999-2004)," Diabetes Care, vol. 30, no. 10, pp. 2517-2522, 2007.

[35] T. E. Matsha, D. J. Soita, M. S. Hassan et al., "Three-year's changes in glucose tolerance status in the Bellville South cohort: rates and phenotypes associated with progression," Diabetes Research and Clinical Practice, vol. 99, no. 2, pp. 223-230, 2013.

[36] J. R. Hebert, L. Clemow, L. Pbert, I. S. Ockene, and J. K. Ockene, "Social desirability bias in dietary self-report may compromise the validity of dietary intake measures," International Journal of Epidemiology, vol. 24, no. 2, pp. 389398, 1995.

[37] L. S. Freedman, A. Schatzkin, D. Midthune, and V. Kipnis, "Dealing with dietary measurement error in nutritional cohort studies," Journal of the National Cancer Institute, vol. 103, no. 14, pp. 1086-1092, 2011.

[38] N. Stefan, H. Staiger, R. Wagner et al., “A high-risk phenotype associates with reduced improvement in glycaemia during a lifestyle intervention in prediabetes," Diabetologia, vol. 58, no. 12, pp. 2877-2884, 2015.

[39] N. Stefan, A. Fritsche, F. Schick, and H. U. Haring, "Phenotypes of prediabetes and stratification of cardiometabolic risk," The Lancet Diabetes and Endocrinology, vol. 4, no. 9, pp. 789798, 2016.

[40] V. Schmid, R. Wagner, C. Sailer et al., "Non-alcoholic fatty liver disease and impaired proinsulin conversion as newly identified predictors of the long-term non-response to a 
lifestyle intervention for diabetes prevention: results from the TULIP study," Diabetologia, vol. 60, no. 12, pp. 23412351, 2017.

[41] M. Hunger, R. Holle, C. Meisinger, W. Rathmann, A. Peters, and M. Schunk, "Longitudinal changes in health-related quality of life in normal glucose tolerance, prediabetes and type 2 diabetes: results from the KORA S4/F4 cohort study," Quality of Life Research, vol. 23, no. 9, pp. 2515-2520, 2014.

[42] K. C. B. Tan, N. M. S. Wat, S. C. F. Tam, E. D. Janus, T. H. Lam, and K. S. L. Lam, "C-reactive protein predicts the deterioration of glycemia in Chinese subjects with impaired glucose tolerance," Diabetes Care, vol. 26, no. 8, pp. 2323-2328, 2003.

[43] E. Selvin, M. W. Steffes, E. Gregg, F. L. Brancati, and J. Coresh, "Performance of A1C for the classification and prediction of diabetes," Diabetes Care, vol. 34, no. 1, pp. 84-89, 2010.

[44] N. Abate and M. Chandalia, "The impact of ethnicity on type 2 diabetes," Journal of Diabetes and its Complications, vol. 17, no. 1, pp. 39-58, 2003.

[45] L. Perreault, Q. Pan, K. J. Mather et al., "Effect of regression from prediabetes to normal glucose regulation on long-term reduction in diabetes risk: results from the Diabetes Prevention Program Outcomes Study," Lancet, vol. 379, no. 9833, pp. 2243-2251, 2012.

[46] M. E. Bowen, "Rethinking diabetes screening and case finding strategies in clinical practice: who's really at risk?," 2016, https://cme.utsouthwestern.edu/sites/cme.utsouthwestern .edu/files/em1608e_082616_protocol_bowen.pdf. 


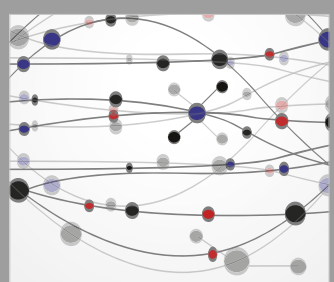

The Scientific World Journal
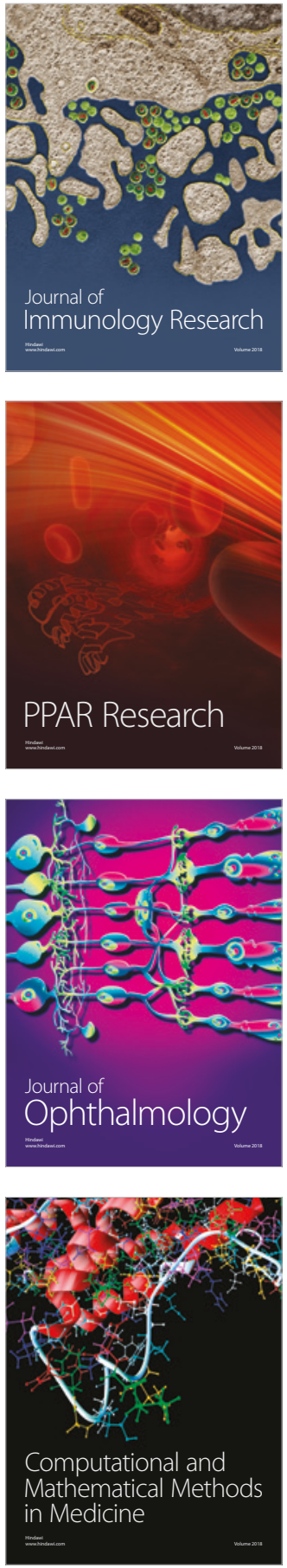

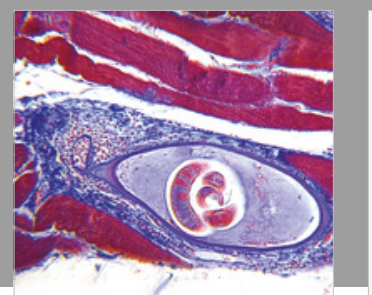

Gastroenterology Research and Practice

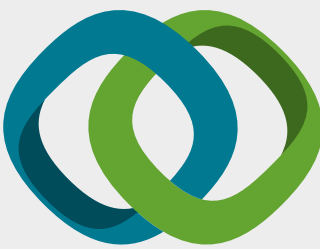

\section{Hindawi}

Submit your manuscripts at

www.hindawi.com
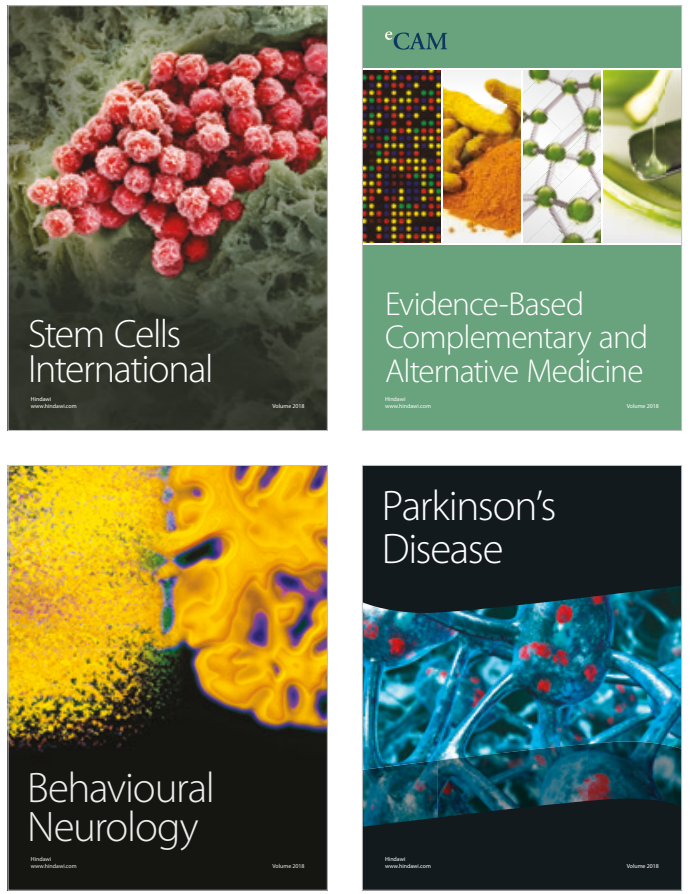

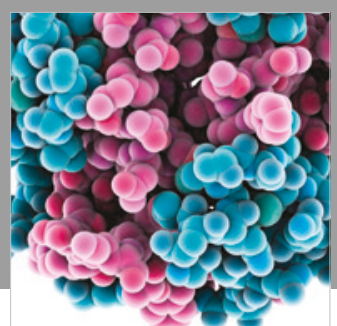

ournal of

Diabetes Research

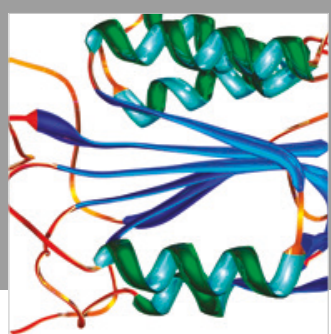

Disease Markers
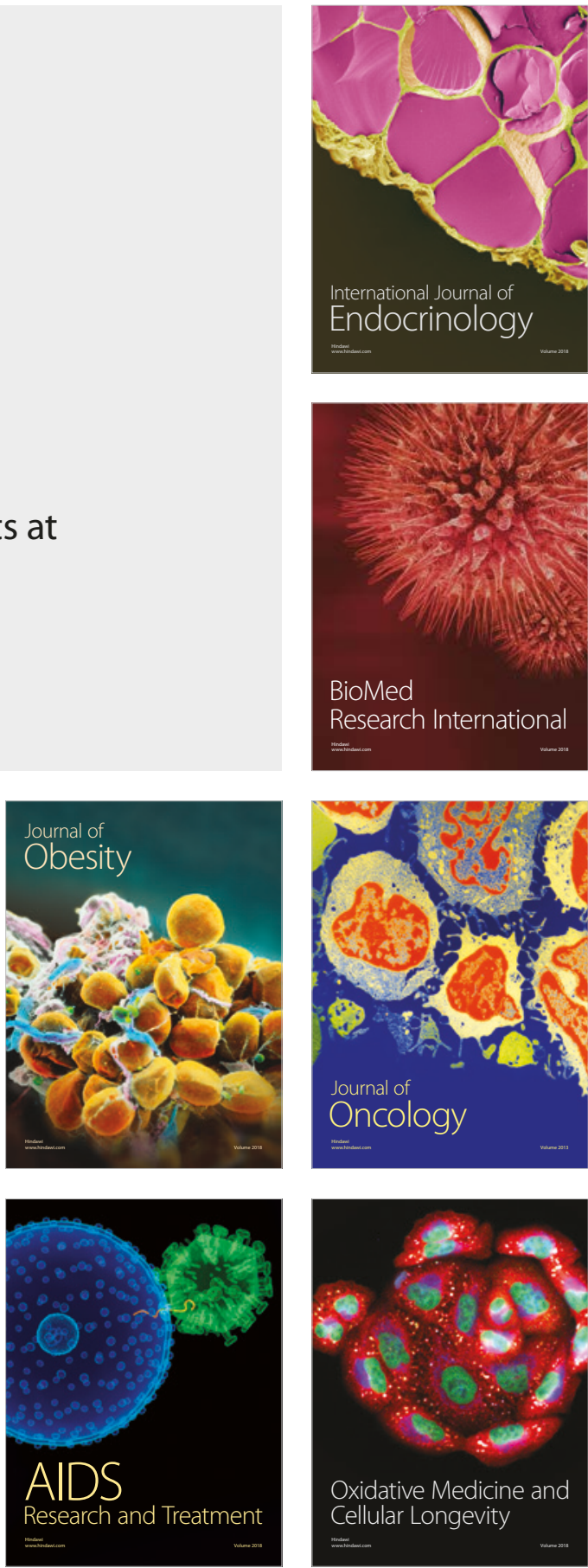